Draft Version July 4, 2018

Preprint typeset using $\mathrm{LAT}_{\mathrm{E}} \mathrm{X}$ style emulateapj v. 5/2/11

\title{
THE MASS AND THE RADIUS OF THE NEUTRON STAR IN THE TRANSIENT LOW MASS X-RAY BINARY
} SAX J1748.9-2021

\author{
Tolga Güver ${ }^{1}$, Feryal Özel ${ }^{2,3}$ \\ Draft version July 4, 2018
}

\begin{abstract}
We use time resolved spectroscopy of thermonuclear X-ray bursts observed from SAX J1748.9-2021 to infer the mass and the radius of the neutron star in the binary. Four X-ray bursts observed from the source with RXTE enable us to measure the angular size and the Eddington limit on the neutron star surface. Combined with a distance measurement to the globular cluster NGC 6440, in which SAX J1748.9-2021 resides, we obtain two solutions for the neutron star radius and mass, $R=8.18 \pm 1.62 \mathrm{~km}$ and $M=1.78 \pm 0.3 \mathrm{M}_{\odot}$ or $R=10.93 \pm 2.09 \mathrm{~km}$ and $M=1.33 \pm 0.33 \mathrm{M}_{\odot}$.

Subject headings: stars: neutron - X-ray: individual: SAX J1748.9-2021
\end{abstract}

\section{INTRODUCTION}

The transient neutron-star X-ray binary SAX J1748.9-2021 located in the globular cluster NGC 6440 was discovered with BeppoSAX during an outburst in 1998 (in't Zand et al. 1999). The identification of the optical and quiescent counterparts of the binary followed shortly afterwards (in't Zand et al. 2001a). In 2001 and 2005, further outbursts were observed (in't Zand et al. 2001b; Markwardt \& Swank 2005), during which a number of X-ray bursts were detected from SAX J1748.9-2021. A total of 16 X-ray bursts that were observed with the Rossi X-ray Timing Explorer (RXTE) showed characteristics of thermonuclear flashes on the neutron star surface (Galloway et al. 2008). In this paper, we use the time-resolved spectroscopy of a subsample of these thermonuclear bursts to determine the mass and radius of the neutron star in SAX J1748.9-2021.

Thermonuclear X-ray bursts are caused by the unstable nuclear burning of matter accreted onto the surface of the neutron star from its companion star. Helium flashes that spread across the neutron star surface give rise to the typical sudden rise in the X-ray flux accompanied by a rise in the temperature. In the cooling phases, the flux and the temperature decrease while the apparent emitting area remains nearly constant (see Lewin et al. 1993 for a review). A recent analysis by Güver et al. (2012a) showed that repeated bursts from the same source give rise to highly reproducible emitting areas, strongly supporting the hypothesis that the entire neutron star surface is emitting during the cooling phase. Because of this, the cooling tails of X-ray bursts can be analyzed to measure the angular size of the neutron star, which can then be converted into an apparent radius using the distance to the source.

A subset of the brightest bursts show Photospheric Radius Expansion (PRE), in which the radiative flux exceeds the local Eddington limit, causing the photosphere

\footnotetext{
${ }^{1}$ Faculty of Engineering and Natural Sciences, Sabanc1 University, Orhanl, Tuzla, Istanbul 34956, Turkey

${ }^{2}$ University of Arizona, Department of Astronomy, $933 \mathrm{~N}$. Cherry Ave., Tucson, AZ 85721

${ }^{3}$ Radcliffe Institute for Advanced Study, Harvard University, 8 Garden St., Cambridge, MA 02138
}

to expand above the stellar surface (see, e.g., Lewin et al. 1993). This results in a characteristic evolution of the temperature and the inferred emitting area during the burst, which can be identified using high time resolution spectroscopy. The Eddington limit on the stellar surface can then be determined from the PRE events by measuring the flux during the expansion and the contraction of the photosphere (Damen et al. 1990).

Thermonuclear bursts and PRE events are useful for measuring the masses and radii of neutron stars (see, e.g., Lewin et al. 1993; Damen et al. 1990; Kuulkers et al. 2003; Özel 2006). In particular, a combination of the apparent angular size obtained during the cooling tails of X-ray bursts, the Eddington limit obtained from the PRE events, and a measurement of the source distance can lead to unique and uncorrelated uncertainties in the stellar mass $M$ and radius $R$.

A rich burst database consisting of 1187 X-ray bursts observed from 48 neutron-star X-ray binaries was made available by more than 10 years of RXTE observations (Galloway et al. 2008). The high signal-to-noise ratio, high-time resolution data allowed Özel et al. (2009), Güver et al. (2010a, b), and Özel et al. (2012a) to measure the masses and radii of four bursting neutron stars and to put strong observational constraints on the equation of state cold matter at supranuclear densities for the first time (Özel, Baym, Güver 2010; see also Steiner, Lattimer \& Brown 2010). In addition, the frequent recurrence of X-ray bursts in a large number of neutron-star X-ray binaries also allowed a systematic study of the reproducibility and the uncertainties in the angular sizes and the Eddington limits of neutron stars measured using these data sets (Güver et al. 2012a, b). These studies, which included all X-ray bursters with two or more PRE events, showed that systematic uncertainties in the two spectroscopic quantities of interest are limited to $10 \%$ in the whole sample.

Following the earlier analyses, we report here the measurement of the mass and the radius of the neutron star in the transient X-ray binary SAX J1748.9-2021. We use the results of Güver et al. (2012a,b) on four X-ray bursts, two of which show evidence of PRE, observed from the source during the 2001 outburst. (See the discussion in that paper and in Section 2 for the burst selection crite- 
ria).

The globular cluster NGC 6440, which hosts SAX J1748.9-2021, is a massive and old Galactic bulge globular cluster with an age of $11_{-2}^{+3}$ Gyr (Origlia et al. 2008). Most of the properties of NGC 6440, such as its metallicity and age, are similar to those of 47 Tucanae, which has been used as a reference for globular cluster distance measurements (see, e.g., Valenti et al. 2007). NGC 6440 is known to host other X-ray sources (see, e.g., Pooley et al. 2002), which includes at least one other transient low-mass X-ray binary (Heinke et al. 2010) and five radio millisecond pulsars (Freire et al. 2008). The distance to the cluster is well constrained; earlier optical observations yield $8.4_{-1.3}^{+1.5} \mathrm{kpc}$ (Kuulkers 2003) while the near-IR studies give $\sim 8.2 \mathrm{kpc}$ (Valenti et al. 2007).

In Section 2, we present the angular size and the Eddington limit from time resolved X-ray spectral analysis. In Section 3, we make use of these along with the distance to NGC 6440 to find two possible solutions for the radius and mass of the neutron star in SAX J1748.9-2021: $R=10.93 \pm 2.09 \mathrm{~km}$ and $M=1.33 \pm 0.33 \mathrm{M}_{\odot}$ or $R=8.18 \pm 1.62 \mathrm{~km}$ and $M=1.78 \pm 0.30 \mathrm{M}_{\odot}$. In Section 4 , we present the existing data on the neutron star obtained during quiescence and discuss the implications of these measurements.

\section{THERMONUCLEAR BURSTS FROM SAX J1748.9-2021}

We base our analysis on the data and methods presented in Güver et al. (2012a, b). Those studies analyzed time resolved spectra obtained by RXTE during thermonuclear bursts from all neutron star X-ray binaries that showed two or more PRE events, including SAX J1748.9-2021.

RXTE observed SAX J1748.9-2021 for a total of $149 \mathrm{ks}$ up to June 2007 (Galloway et al. 2008). In these observations, a total of 16 thermonuclear bursts were detected, all of which were within an eight day period during the 2001 outburst of the binary. Because of the increased persistent flux levels during the outburst, all but four of the observed bursts occurred when the persistent flux was higher than $10 \%$ of the peak burst flux (determined in accordance with the parameter $\Gamma$ in Galloway et al. 2008). When the persistent flux is high, subtracting the background accretion flux from the burst spectra introduces systematic uncertainties in the measured surface areas and Eddington fluxes. Therefore, as with all our previous studies, we exclude from the analysis the X-ray bursts that have $\Gamma>10 \%$.

In Güver et al. (2012a), we analyzed the spectra during the cooling tails of these four bursts, measured the most likely angular size of the neutron star, and quantified any systematic uncertainties arising from possible spectral evolution, inhomogeneous burning, rotational broadening, etc. The cooling tails of the X-ray bursts are shown in Figure 1. Assuming a Gaussian probability distribution for the apparent angular size, we found that the most likely value is $A=89.7(\mathrm{~km} / 10 \mathrm{kpc})^{2}$ with a standard deviation of $9.6(\mathrm{~km} / 10 \mathrm{kpc})^{2}$.

In the burst sample, two events showed evidence of photospheric radius expansion. We measured the Eddington flux at the touchdown moment for these two events (Güver et al. 2012b), which we present in Table 1. We also show in Figure 2 the confidence contours of the

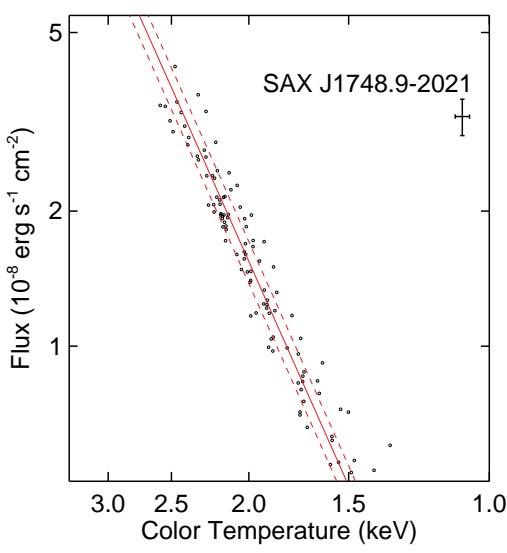

FIG. 1.- Spectral evolution during the cooling tails of the X-ray bursts observed from SAX J1748.9-2021. Red solid and dashed lines correspond to the most likely value of the apparent radius and to its systematic uncertainty and follow $F \propto T^{4}$ relation.

TABLE 1

Touchdown Flux Values.

\begin{tabular}{ccc}
\hline \hline Burst ID & MJD & $\begin{array}{c}\text { Touchdown Flux } \\
\left(10^{-8} \mathrm{erg} \mathrm{s}^{-1} \mathrm{~cm}^{-2}\right)\end{array}$ \\
\hline 1 & 52190.38947 & $4.52 \pm 0.14$ \\
2 & 52190.46882 & $3.54 \pm 0.12$ \\
\hline
\end{tabular}

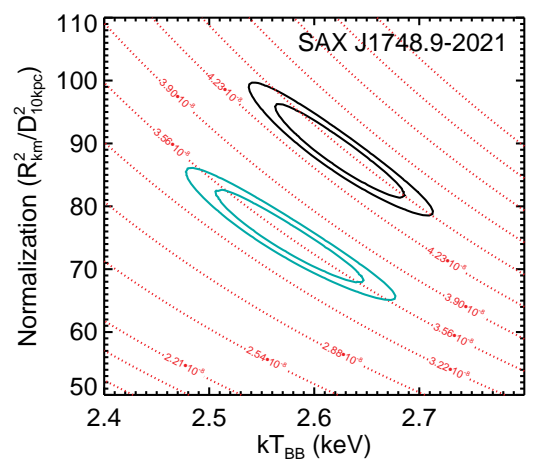

FIG. 2.- $68 \%$ and $90 \%$ confidence contours of the blackbody normalization and color temperature at the touchdown moment from two X-ray bursts that show evidence of photospheric radius expansion.

spectral parameters at the touchdown moment for each burst. The fact that there are only two PRE events prevents us from quantifying the level of systematic uncertainty in the Eddington flux from these two bursts alone. However, combined with the sample of other sources that showed only a couple of PRE bursts, we were able to show statistically that the most likely systematic uncertainty in the Eddington flux of SAX J1748.9-2021 is equal to $11 \%$ (see Figures 10 and 11 in Section 6 of Güver et al. 2012 b). For this reason, we adopt a Gaussian probability distribution for the Eddington flux of SAX J1748.9-2021 centered at $F_{\text {Edd }}=4.03 \times 10^{-8} \mathrm{erg} \mathrm{s}^{-1} \mathrm{~cm}^{-2}$ and with a width of $0.44 \times 10^{-8} \mathrm{erg} \mathrm{s}^{-1} \mathrm{~cm}^{-2}$.

There are sources of possible systematic uncertainties 
in addition to those discussed in Güver et al. (2012a,b) in the measurements of the apparent angular size and the Eddington flux from thermonuclear bursts. For example, uncertainties may arise from anisotropy in the bursts, as noted by Zamfir et al. (2012) for the Xray binary GS $1826-24$. This is unlikely to apply to SAX J1748.9-2021 given the measured 5\% systematic variation in the radius of the emitting region. A second uncertainty comes from the absolute calibration of the fluxes measured by X-ray satellites. Cross calibration of RXTE with other X-ray satellites help in mitigating this uncertainty but do not eliminate it (see Jahoda et al. 2006 and Steiner et al. 2010b). Finally, we make here the assumption that the burst flux at the touchdown moment in PRE bursts corresponds to the Eddington flux on the neutron star surface. Steiner et al. (2010a) considered the possibility that the photosphere remains above the neutron star surface at "touchdown" and discussed uncertainties in the radius measurements that may arise from relaxing this assumption.

\section{THE MASS AND RADIUS OF SAX J1748.9-2021}

The first measurement of the distance to the globular cluster NGC 6440, which was carried out in the optical wavebands, yielded a distance of $8.4_{-1.3}^{+1.5} \mathrm{kpc}$ (Kuulkers et al. 2003). A more recent study by Valenti et al. (2007) in the near-IR obtained the cluster distance by comparing the luminosity function and color-magnitude diagrams with a reference cluster, 47 Tucanae. They found a distance of $8.2 \pm 0.6 \mathrm{kpc}$, which is consistent with the earlier measurement but has an improved uncertainty, and where the quoted error takes into account the systematic uncertainties introduced by the method of comparing to the reference cluster. In the following, we adopt this latter distance and its uncertainty.

To determine the mass and the radius of the neutron star in SAX J1748.9-2021, we follow the Bayesian method discussed in detail in Özel et al. (2009, 2012a). For each observable, we assign an independent probability distribution function. For the touchdown flux $P\left(F_{T D}\right) d F_{T D}$, the apparent angular size $P(A) d A$, and the distance $P(D) d D$, we use Gaussian probability distribution functions with the parameters discussed above.

There are two other model parameters that are needed to convert the observables to a measurement of the neutron star mass and radius: the hydrogen mass fraction $X$ in the atmosphere and the color correction factor $f_{c}$. The latter is used to convert the color temperatures obtained from blackbody fits to X-ray spectra to the effective temperature of the neutron star atmosphere. In the absence of any prior knowledge, we assume for the hydrogen mass fraction $X$ a box-car distribution between the entire range of possible values of 0.0 and 0.7 . The color correction factor obtained from model atmospheres was discussed in detail in Güver et al. (2010a, b, 2012a). For this model parameter, we adopt a box-car distribution in the range $f_{c}=1.35 \pm 0.05$ as in the earlier studies that is appropriate for the fluxes observed in the cooling tails of the bursts from SAX J1748.9-2021.

We convert the likelihoods over the touchdown flux, the apparent angular size, and the distance to one over the neutron star mass, radius, and distance following Özel et al. (2012a) and integrate over the distance like- lihood. The resulting probability distribution over the neutron star mass and radius peaks in two close regions in the mass-radius space, as shown in Figure 3. Even though we calculate the full probability distribution over these parameters, we show with filled contours the regions above $M>1.2 M_{\odot}$ in Figure 3, which are astrophysically more relevant. This is supported both observationally, as the vast majority of measurements point to neutron star masses above this limit, and by studies of pre-supernova stellar cores, which are expected to leave behind neutron stars with birth masses $\gtrsim 1.1 M_{\odot}$ (see Özel et al. 2012b for a discussion of all the current measurements and the theoretical expectations).

To obtain the individual measurements of the stellar radius and mass, we marginalize the two-dimensional probability distribution over mass and radius, respectively. We find that the radius and mass of the neutron star is either $R=8.18 \pm 1.62 \mathrm{~km}$ and $M=1.78 \pm 0.30 \mathrm{M}_{\odot}$ or $R=10.93 \pm 2.09 \mathrm{~km}$ and $M=1.33 \pm 0.33 \mathrm{M}_{\odot}$, where the uncertainties represent the $1 \sigma$ width of the best-fit Gaussians to the probability density functions over the stellar radius and mass.

\section{DISCUSSION}

Spectroscopic measurements of neutron star radii have been carried out to date using several techniques. The first method, which consists of the analysis of thermonuclear bursts that we used in the present paper, has previously been applied to four neutron star binaries: EXO 1745-248 (Özel et al. 2009), 4U 1608-52 (Güver et a. 2010a), 4U 1820-30 (Güver et al. 2010b), and KS 1731-260 (Özel et al. 2012a). Using the evolution of burst spectra as a function of burst flux, Zamfir et al. (2012) carried out a radius measurement in GS 1826-24. A second method that utilizes the thermal X-ray emission from neutron star binaries during quiescence has yielded radius measurements for four additional sources: the neutron stars in M13, NGC 2808, and $\omega$ Cen (Webb \& Barret 2007) as well as U24 in NGC 6397 (Guillot et al. 2011; see the compilation in Özel (2013) for a detailed discussion of each source and the corresponding measurement uncertainties as well as for radii measurements in isolated neutron stars). The mass and radius we find here for the neutron star in SAX J1748.9-2021 is in good agreement with these previous measurements. Even though no single measurement currently constrains at a significant level the neutron star equation of state, collectively, they point to neutron star radii that are in the $8-11 \mathrm{~km}$ range, which is consistent only with a subset of the proposed equations of state (see Özel, Baym, \& Güver 2010).

The two techniques mentioned above have never been applied to the same source, which would provide an important test for the methods' consistency. For that purpose, X-ray bursters that are also transient sources, such as SAX J1748.9-2021, can be very useful. SAX J1748.9-2021 has been in quiescence since its last outburst in 2005. During this time interval, the Chandra X-ray Observatory observed the globular cluster NGC 6440 one time in 2009 with an exposure of approximately $50 \mathrm{ks}$. We analyzed the data obtained during the observation using standard Chandra data analy- 


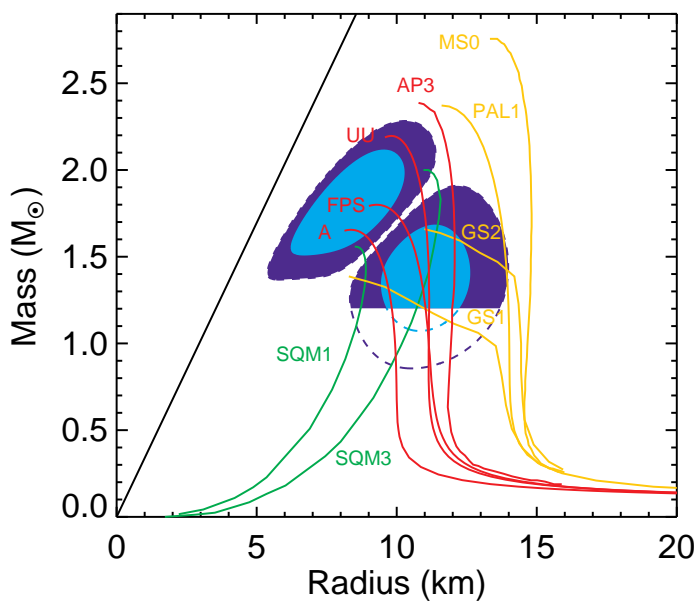

FIG. 3.- Dashed lines show the $68 \%$ and $95 \%$ confidence contours for the mass and the radius of the neutron star in SAX J1748.9-2021. Filled regions show the more astrophysically relevant regions, with $\mathrm{M}>1.2 \mathrm{M} \odot$.

sis techniques 4 Although the source is clearly detected, with only about 260 source counts, it is not possible to obtain any reliable constraints on the apparent angular size of the neutron star. However, unlike some other transients (e.g., Cen X-4, Cackett et al. 2010), the existing X-ray spectrum shows no sign of a non-thermal component that would indicate some residual accretion. In that optimal case, a new deep Chandra observation will provide the opportunity to obtain an independent constraint on the radius of this neutron star, and hence a direct comparison of the results of the two methods.
We thank Elena Valenti for very useful discussions on the measurements of the distances to globular clusters. This work was supported by NASA ADAP grant NNX10AE89G and in part by the Radcliffe Institute for Advanced Study at Harvard University. This research has made use of data obtained from the High Energy Astrophysics Science Archive Research Center (HEASARC), provided by NASA's Goddard Space Flight Center.

\section{REFERENCES}

Cackett, E. M., Brown, E. F., Miller, J. M., \& Wijnands, R. 2010, ApJ, 720, 1325

Damen, E., Magnier, E., Lewin, W. H. G., Tan, J., Penninx, W., \& van Paradijs, J. 1990, A\&A, 237, 103

Freire, P. C. C., Ransom, S. M., Bégin, S., Stairs, I. H., Hessels, J. W. T., Frey, L. H., \& Camilo, F. 2008, ApJ, 675, 670

Galloway, D. K., Muno, M. P., Hartman, J. M., Psaltis, D., \& Chakrabarty, D. 2008, ApJS, 179, 360

Guillot, S., Rutledge, R. E., \& Brown, E. F. 2011, ApJ, 732, 88

Güver, T., Özel, F., Cabrera-Lavers, A., \& Wroblewski, P. 2010a, ApJ, 712, 964

Güver, T., Wroblewski, P., Camarota, L., Özel, F. 2010b, ApJ, 719,1807

Güver, T., Özel, F., \& Psaltis, D. 2012a, ApJ, 747, 77

Güver, T., Psaltis, D., Oüzel, F. 2012b, ApJ, 747, 76

Heinke, C. O., et al. 2010, ApJ, 714, 894

in 't Zand, J. J. M., et al. 1999, A\&A, 345, 100

in't Zand, J. J. M., van Kerkwijk, M. H., Pooley, D., Verbunt, F., Wijnands, R., \& Lewin, W. H. G. 2001a, ApJ, 563, L41

in't Zand, J., Pooley, D., Lewin, W., van Kerkwijk, M., \& Verbunt, F. 2001b, The Astronomer's Telegram, 74, 1

Jahoda, K., Markwardt, C. B., Radeva, Y., et al. 2006, ApJS, 163, 401

Kuulkers, E., den Hartog, P. R., in't Zand, J. J. M., Verbunt, F. W. M., Harris, W. E., \& Cocchi, M. 2003, A\&A, 399, 663
Lewin, W. H. G., van Paradijs, J., \& Taam, R. E. 1993, Space Sci. Rev., 62, 223

Markwardt, C. B., \& Swank, J. H. 2005, The Astronomer's Telegram, 495, 1

Özel, F. 2006, Nature, 441, 1115

Origlia, L., Lena, S., Diolaiti, E., Ferraro, F. R., Valenti, E., Fabbri, S., \& Beccari, G. 2008, ApJ, 687, L79

Özel, F., Güver, T., \& Psaltis, D. 2009, ApJ, 693, 1775

Özel, F., Baym, \& Güver, T. 2010, Phys. Rev. D, 82, 101301

Özel, F., Gould, A., \& Güver, T. 2012a, ApJ, 748, 5

Özel, F., Psaltis, D., Narayan, R., \& Santos Villarreal, A. 2012b, ApJ, 757, 55

Özel, F. 2013, Rep. Prog. Phys. 76, 016901

Pooley, D., et al. 2002, ApJ, 573, 184

Steiner, A. W., Lattimer, J. M., \& Brown, E. F. 2010a, ApJ, 722, 33

Steiner, J. F., McClintock, J. E., Remillard, R. A., et al. 2010b, ApJ, 718, L117

Valenti, E., Ferraro, F. R., \& Origlia, L. 2007, AJ, 133, 1287

Webb, N. A., \& Barret, D. 2007, ApJ, 671, 727

Zamfir, M., Cumming, A., \& Galloway, D. K. 2012, ApJ, 749, 69

\footnotetext{
${ }^{4}$ http://cxc.cfa.harvard.edu/ciao/
} 